\title{
Angra dos Reis Reactor Neutrino Oscillation Experiment
}

\author{
J. C. Anjos ${ }^{1}$, A. F. Barbosa ${ }^{1}$, A. Bernstein ${ }^{2}$, N. S. Bowden ${ }^{3}$, W. Fulgione ${ }^{4}$, E. Kemp ${ }^{5}$, J. Magnin ${ }^{1}$, H. \\ Nunokawa $^{6}$, O. L. G. Peres ${ }^{5}$, D. Reyna ${ }^{7}$, A. Schilithz ${ }^{1}$, R. C. Shellard ${ }^{1,6}$, and and R. Zukanovich Funchal ${ }^{8}$ \\ (1)Centro Brasileiro de Pesquisas Físicas, Rua Dr. Xavier Sigaud 150, Rio de Janeiro, RJ, 22290-180, Brazil \\ (2)Lawrence Livermore National Laboratory, 7000 East Ave, Livermore, CA 94551, USA \\ (3)Sandia National Laboratories, 7011 East Ave, Livermore, CA 94550, USA \\ (4)Istituto Nazionale di Astrofisica - Istituto di Fisica dello Spazio Interplanetario, Corso Fiume 4, 10100, Torino, Italy \\ (5)Instituto de Física Gleb Wataghin, Universidade Estadual de Campinas, \\ Caixa Postal 6165, Campinas, SP, 13083-970, Brazil \\ (6)Departamento de Física, Pontifícia Universidade Católica do Rio de Janeiro, \\ Caixa Postal 38071, Rio de Janeiro, RJ, 22452-970, Brazil \\ (7)Argonne National Laboratory, 9700 S. Cass Avenue, Argonne, IL, 60439, USA and \\ (8)Instituto de Física, Universidade de São Paulo, Caixa Postal 66318 São Paulo, SP, 05315-970, Brazil
}

\section{Received on 10 August, 2006}

\begin{abstract}
We present the status and plans of the Angra Project, a new reactor neutrino oscillation experiment, proposed to be built in Brazil at the Angra dos Reis nuclear complex. This experiment is aimed to measure $\theta_{13}$, the last unknown of the three neutrino mixing angles. We propose a high sensitivity multi-detector experiment, able to reach a sensitivity to antineutrino disappearance down to $\sin ^{2} 2 \theta_{13}=0.006$ in a three years running period, by combining a high luminosity design, very low background from cosmic rays and careful control of systematic errors. We also intend to explore the possibility to use the neutrino detector for purposes of safeguards and non-proliferation of nuclear weapons.
\end{abstract}

Keywords: Reactor Neutrinos; Neutrino Oscillations; Neutrino Mixing Angle; Safeguards and Non-Proliferation

\section{INTRODUCTION}

Nuclear reactors played a very important role in neutrino physics. Indeed, neutrinos were first experimentally detected 50 years ago by Reines and Cowan [1] using the outcoming $\bar{v}_{e}$ flux from a nuclear reactor and observing neutrino interactions through the inverse- $\beta$ decay,

$$
\overline{\mathrm{v}}_{e}+p \rightarrow e^{+}+n
$$

Recently, the KamLAND experiment [2], also using neutrinos produced in nuclear reactors, observed clear signals of neutrino oscillation, establishing the Mikheyev-SmirnovWolfenstein (MSW) [3] large mixing angle solution for the solar neutrino problem.

Despite the fact that enormous progress has been made in neutrino physics in the last decade [4], there are still three unknown parameters necessary to fully describe the neutrino oscillation phenomena: the mixing angle $\theta_{13}$, the CP phase $\delta$ and the sign of the mass squared difference, $\Delta m_{13}^{2} \equiv m_{3}^{2}-m_{1}^{2}$, which is positive (negative) if the mass hierarchy is normal (inverted).

There is a general consensus that the measurement of $\theta_{13}$ is the most important step towards further progress in the field. In fact, $\theta_{13}$ controls the experimental accessibility of the other two unknown parameters. If $\theta_{13}$ is non-zero and relatively large, it can open the possibility to measure $\delta$ by the observation of $\mathrm{CP}$ violation in the leptonic sector and to determine the neutrino mass hierarchy.

While it is true that $\theta_{13}$ can also be measured in accelerator based appearance experiments, such measurements are subject to degeneracies [5] due to specific combinations of the unknown oscillation parameters. On the other hand, reactor neutrino short baseline experiments can provide a very clean measurement of $\theta_{13}$ [6], free of ambiguities from degeneracies and matter effects [3]. Moreover, by combining the reactor measurement of $\theta_{13}$ and accelerator based oscillation experiments, the determination of the other mixing parameters become significantly improved [7].

In this contribution we describe the main features of the Angra Experiment [8], a project to observe antineutrino disappearance at the Angra dos Reis nuclear complex in Brazil. The experimental approach is to measure, with high precision, the neutrino energy spectra at two different distances from the reactor core: one of them obtained by a close detector (hereafter called the Near Detector - ND) located at $\sim 300 \mathrm{~m}$ from the reactor, providing us with the reference spectrum minimally affected by oscillations; and the other one obtained by a detector placed at a suitable distance, typically $\sim 1.5 \mathrm{~km}$ (the Far Detector - FD), to observe the spectral distortion induced by oscillations. Thus, the neutrino mixing parameter values can be inferred by the differences between the ND and FD spectral shapes.

As a first step of the experiment, we plan to build a small detector with a baseline $L \simeq 60-70 \mathrm{~m}$, which will be used to measure the initial neutrino flux at the production point, practically not affected by oscillations, as well as the reference energy spectrum. This detector technology may have applications on the implementation of nuclear safeguards in the context of the Nuclear Non-Proliferation Treaty. Moreover, such a Very Near Detector (VND) will work as a prototype to test concepts and elements of the larger ND and FD and to perform general cross-checks. 


\section{ANGRA DOS REIS: THE NUCLEAR REACTOR COMPLEX MAIN FEATURES}

The Brazilian nuclear reactors are located at Angra dos Reis, a city about $150 \mathrm{~km}$ south of Rio de Janeiro. The Angra nuclear reactor complex has two operational reactors (Angra I and II) and a third one under approval process (Angra III, similar to Angra II). The state owned company Eletronuclear is responsible for the general management and commercial operation of the plant. The thermal power of the reactors are $2 \mathrm{GW}$ and $4 \mathrm{GW}$ for Angra I and II, with uptimes around 83\% and $90 \%$, respectively. The topology of the surrounding terrain, formed by mountainous granite, is an advantage of the site.

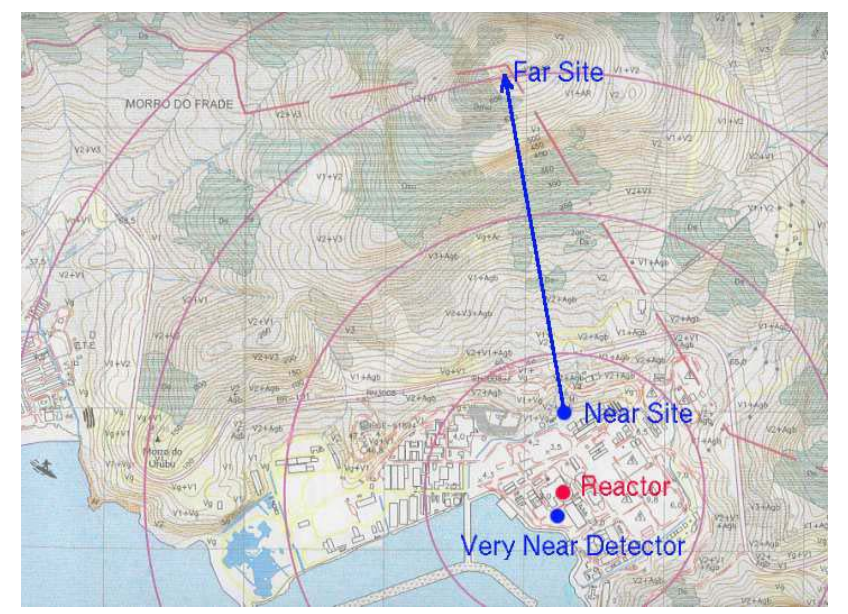

FIG. 1: Map of the Angra site, where the positions of the reactor, VND, ND and FD are indicated. The solid line indicates the horizontal tunnel to be excavated to give access to the far detector experimental hall, under the Morro do Frade peak. The solid circles indicate the distance from the Angra II reactor in $500 \mathrm{~m}$ steps.

We show in Fig. 1 a topographic map of the reactor complex where the positions of the three detectors are indicated. At about $1.5 \mathrm{~km}$ from the Angra II reactor, there is the so-called "Morro do Frade", the highest nearby peak (700 m), providing an overburden of $\sim 2000$ m.w.e.

In Fig. 2 we present the expected muon flux as a function of the depth. As can be seen, the Angra FD site has overburden comparable to dedicated underground facilities. Thus, a substantial reduction of the cosmic ray induced background can be achieved by construction of an underground experimental hall, accessible by a horizontal tunnel to be built at a much lower cost than vertical shafts.

\section{OSCILLATION PROBABILITY}

Neglecting terms that will give very small contributions, the antineutrino survival probability, $P\left(\bar{v}_{e} \rightarrow \bar{v}_{e}\right)$, in vacuum is described by

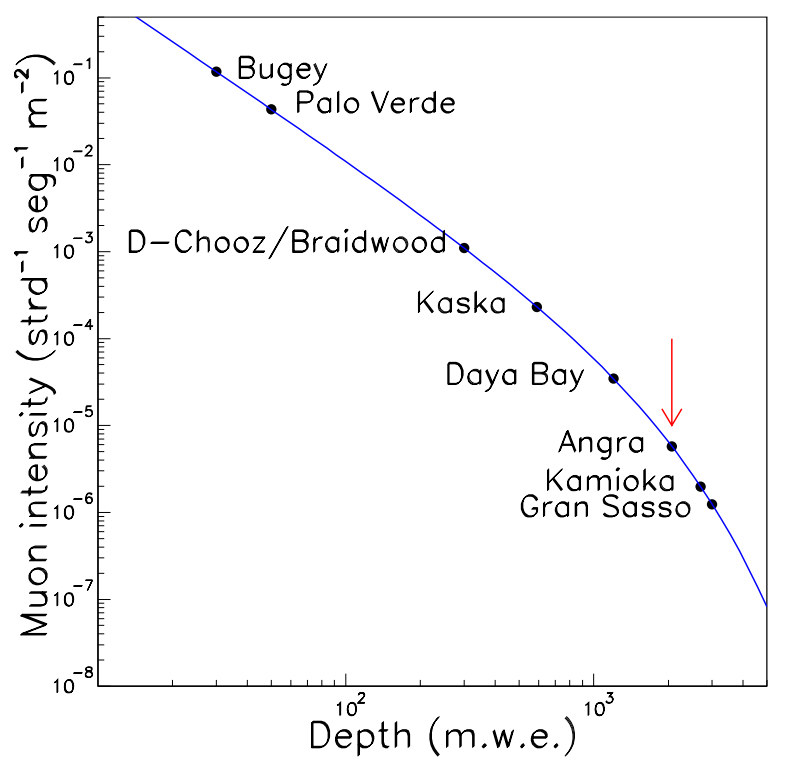

FIG. 2: The solid curves gives the underground muon flux extrapolated from the LVD experiment results [9]. Big dots indicate overburdens of different reactor experiments. The arrow points to the Angra FD situation. The two deepest installations, added for comparison purpose, are dedicated underground laboratories (Kamioka in Japan and Gran Sasso in Italy).

$$
\begin{aligned}
& P\left(\overline{\mathrm{v}}_{e}\right.\left.\rightarrow \overline{\mathrm{v}}_{e}\right) \approx 1-\sin ^{2} 2 \theta_{13} \sin ^{2}\left(\frac{\Delta m_{13}^{2} L}{4 E}\right) \\
&-\cos ^{4} \theta_{13} \sin ^{2} 2 \theta_{12} \sin ^{2}\left(\frac{\Delta m_{12}^{2} L}{4 E}\right),
\end{aligned}
$$

where $L$ is the propagation distance (the baseline).

Taking into account the typical energies for reactor neutri$\operatorname{nos}(E \sim 3-5 \mathrm{MeV})$, the first minimum of the probability, i.e., the point of maximal flavour conversion, can be achieved at $L \sim 1.5 \mathrm{~km}$, coinciding with the proposed far detector baseline.

We show in Fig. 3 a plot of the probability as a function of $L / E$, where relevant limits for the Angra case are indicated by the vertical lines. Here the probability was computed assuming $\sin ^{2} 2 \theta_{13}=0.05$, and using the best fit values of the mixing parameters from solar, atmospheric, reactor and accelerator neutrino measurements, $\Delta m_{12}^{2}=8 \times 10^{-5} \mathrm{eV}^{2}, \sin ^{2} \theta_{12}=$ $0.31, \Delta m_{13}^{2}=2.5 \times 10^{-3} \mathrm{eV}^{2}, \sin ^{2} 2 \theta_{23}=1.0[2,10-13]$.

¿From this plot it is clear that for both near detectors (VND and ND), the oscillation effect is negligible $(P \sim 1)$ whereas the first oscillation minimum occurs at the far detector distance for $E \sim 2-5 \mathrm{MeV}$. Let us note that the third term in Eq. (2) is negligible at the baseline we are considering $(L \sim$ $1.5 \mathrm{~km}$ ), that explains why we can perform a clean measurement of $\theta_{13}$ without suffering from the ambiguities of other mixing parameters and basically free of matter effects. 


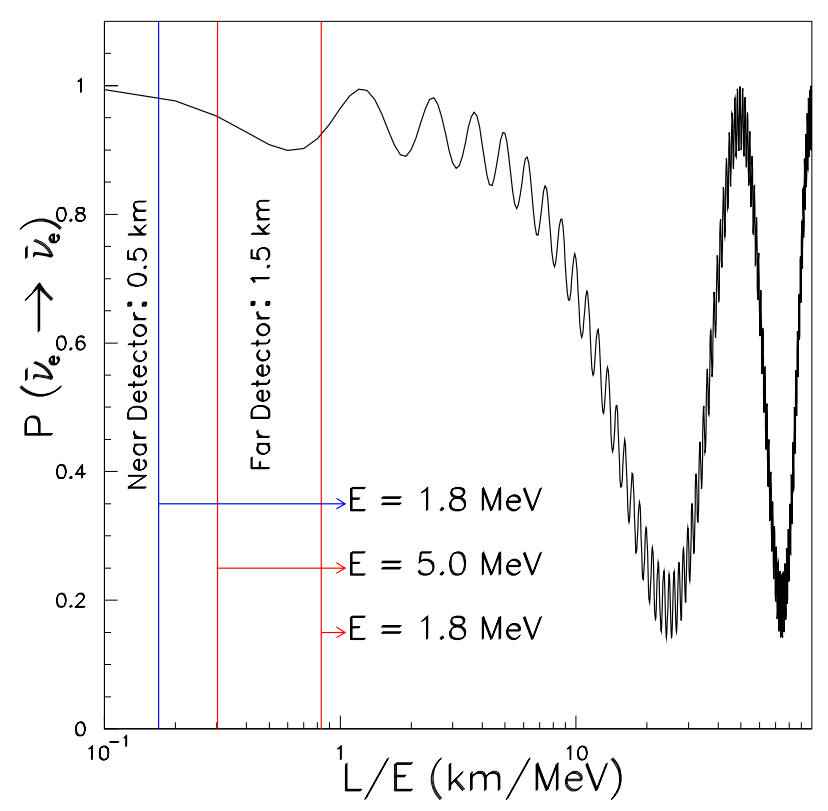

FIG. 3: Antineutrino survival probability $P\left(\bar{v}_{e} \rightarrow \bar{v}_{e}\right)$ as function of the ratio $L / E[\mathrm{~km} / \mathrm{MeV}]$. Vertical lines indicate some relevant reactor neutrino energies. $E=1.8 \mathrm{MeV}$ corresponds to the reaction threshold. The peak of the energy spectrum weighted by the detection cross section in the absence of oscillation is at $E \sim 4 \mathrm{MeV}$, and the contribution of neutrinos with energy $\lesssim 5 \mathrm{MeV}$ is the most important.

\section{THE EXPERIMENTAL DESIGN}

The Angra neutrino oscillation experiment will consist of two neutrino detectors to be set at the near/far configuration, as explained above. The third one, the VND, is planned to be built as a prototype. In addition, the very near detector will work as a non-intrusive nuclear reactor monitor, within the international effort promoted by the IAEA, to develop tools for verification of safeguards and non-proliferation of nuclear weapons. The VND ( 1 ton), ND (50 ton) and FD (500 ton) will have a very similar design, scaled appropriately, as shown in Fig. 4. The design concept is based on two complementary systems: the antineutrino detector and the mиon veto, briefly described in the following sections.

\section{A. The antineutrino detector}

The antineutrino detector, designed in a standard monolithic configuration is made of three concentric volumes with different functionalities: i) the $\bar{v}_{e}$ target - innermost volume filled with gadolinium doped liquid scintillator with high efficiency to tag inverse- $\beta$ decay events due to the high cross section for neutron capture; ii) the gamma catcher - intermediate volume filled with standard scintillator to increase the detection volume for gammas from neutron capture and, as a consequence, the fiducial volume of the target; iii) the nonscintillating buffer - the outermost volume filled with mineral oil to reduce the radioactivity from outside.

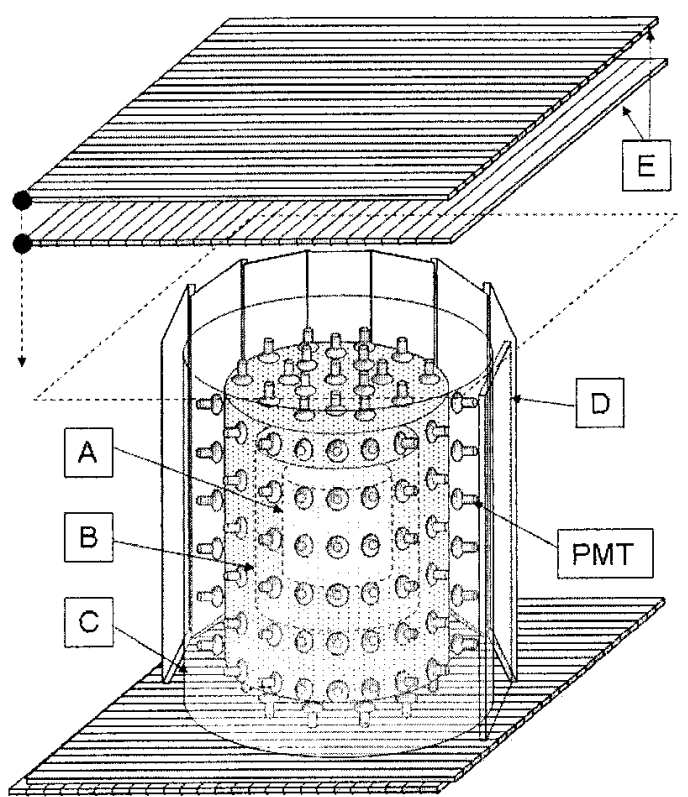

FIG. 4: Schematic view of the detector showing all subsystems, described in detailed in the text. A) Target ; B) Gamma-Catcher ; C) Buffer ; D) Vertical tile of veto system. Some tiles of the frontal part were omitted just to show the antineutrino detection system. E) X-Y horizontal paddles of the Veto system. They are placed above and under the external cylinder of the buffer, for muon tracking through the detector. The top X-Y system in the drawing is shifted upward for viewing purposes. The dashed area shows its true position.

The phototubes are installed in the buffer outer wall, with about $15 \%$ of surface coverage, looking inside to monitor light pulses from interactions. The organic liquid scintillator is a solvent mixture with a basic component like $\mathrm{C}_{n} \mathrm{H}_{2 n+2},\langle n\rangle$ $=9.8$, and additional activators and wavelength shifters such as PPO and POPOP. The proton richness of this kind of compound provides a large number of target particles for the main antineutrino interaction channel, the inverse $\beta$-decay (Eq. (1)). All three volumes are filled with the same liquid basis to guarantee their optical match and uniformity of light collection by the phototubes.

The $\bar{v}_{e}$ signature is given by the detection in time coincidence of the secondary particles: $e^{+}, n$. The positron generates the prompt signal and the delayed detection of gammas from neutron capture gives the second signal. The gamma detection must occur in an adjustable time window with typical duration of $3 \tau<\Delta t<5 \tau$, where $\tau$ is the mean time interval for neutron capture. Noise rejection can be considerably improved by reducing the time window for coincident pulses. The neutron capture cross section is increased by a factor up to $10^{5}$ by loading the scintillator with gadolinium in a concentration of $0.1-0.5 \%$, resulting in $15 \mu \mathrm{s}<\tau<50 \mu \mathrm{s}$, a factor $\sim 5$ shorter than the mean time interval for capture on free protons in unloaded scintillators [14].

The neutrino energy is related to the positron energy by $\mathrm{E}_{v}=E_{e^{+}}+\Delta m_{\mathrm{np}}$, i.e. the positron energy plus the neutronproton mass difference $\left(\Delta m_{\mathrm{np}}\right)$. We remind the reader that the 
visible energy is $E_{\mathrm{vis}}=E_{e^{+}}+E_{\gamma}$, with an additional contribution from the photons from the positron annihilation.

\section{B. The muon veto}

The muon veto is an external layer, consisting of plastic scintillator paddles, screening the internal systems (the antineutrino detector). All paddles have attached phototubes at both ends and work in fast time coincidence, triggered by muons from cosmic rays passing through the apparatus.

The muons can produce neutrons by spallation either in the detector or in surrounding material. These background neutrons can be captured in the scintillator, producing gammas in delayed coincidence with the muon signal or any other spurious signal. This kind of events can mimic a true anti-neutrino interaction. So, after a trigger coming from the muon veto system, the readout of the front-end electronics is suppressed in case of an instantaneous recognition of a muon induced event, or otherwise, just flagged to enable an accurate analysis of the background.

Substantial improvement in noise rejection can be achieved with a suitable overburden condition, as planned for the ND and FD experimental rooms. The VND is planned to be installed at least some tens of meters underground, and with an extra shielding, provided by thick walls of some dense material (iron or concrete, for instance).

\section{EXPERIMENTAL REACH}

In Table I, we show preliminary estimations of the signal and background rates per day, assuming target masses of 1 ton, 50 ton and 500 ton for VND $(L=50 \mathrm{~m})$, ND $(L=300$ $\mathrm{m})$, and FD $(L=1500 \mathrm{~m})$, respectively.

\begin{tabular}{lccl}
\hline Detector & Very Near & Near & Far \\
\hline Signal & 1800 & 2500 & 1000 \\
Muons (Hz) & 150 & $\sim 30$ & 0.3 \\
${ }^{9}$ Li bkg & 44 & $\leq 20$ & $\sim 2$ \\
\hline
\end{tabular}

TABLE I: Expected rates per day for the Angra Experiment. Signal and ${ }^{9} \mathrm{Li}$ background (correlated noise) are in events/day units. Calculations were done assuming target masses as follows: 1 ton VND $(L=50 \mathrm{~m}), 50$ ton $\mathrm{ND}(L=300 \mathrm{~m})$ and 500 ton $\mathrm{FD}(L=1500 \mathrm{~m})$.

Based on the yields at the ND and FD, the expected sensitivity on $\sin ^{2} 2 \theta_{13}$ at $90 \%$ CL as a function of the integrated luminosity is shown in Fig. 5. The assumed value of $\Delta m_{13}^{2}$ is $2.5 \times 10^{-3} \mathrm{eV}^{2}$. The calculations were performed by minimizing the $\chi^{2}$ function built to take into account four different types of systematic errors, $\sigma_{i j}$, whose assumed values are indicated in the figure. We used the same $\chi^{2}$ function used in [15]. The subscripts $D(d)$ represents errors correlated (uncorrelated) between detectors and $\mathrm{B}(\mathrm{b})$ errors correlated (uncorrelated) between bins of the measured energy spectra. As can be seen a limit of $\sin ^{2} 2 \theta_{13}=0.006$ at $90 \%$ confidence level can be achievable within three years provided that systematic errors are well controlled.

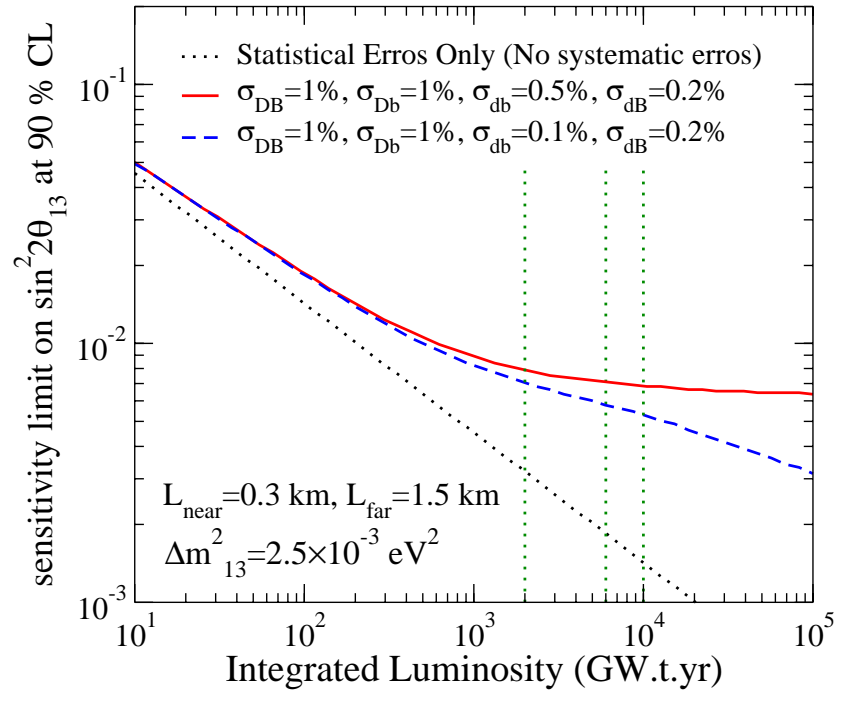

FIG. 5: Sensitivity limit as a function of the integrated luminosity. Vertical dashed lines represent 1, 3 and 5 years of data taking.

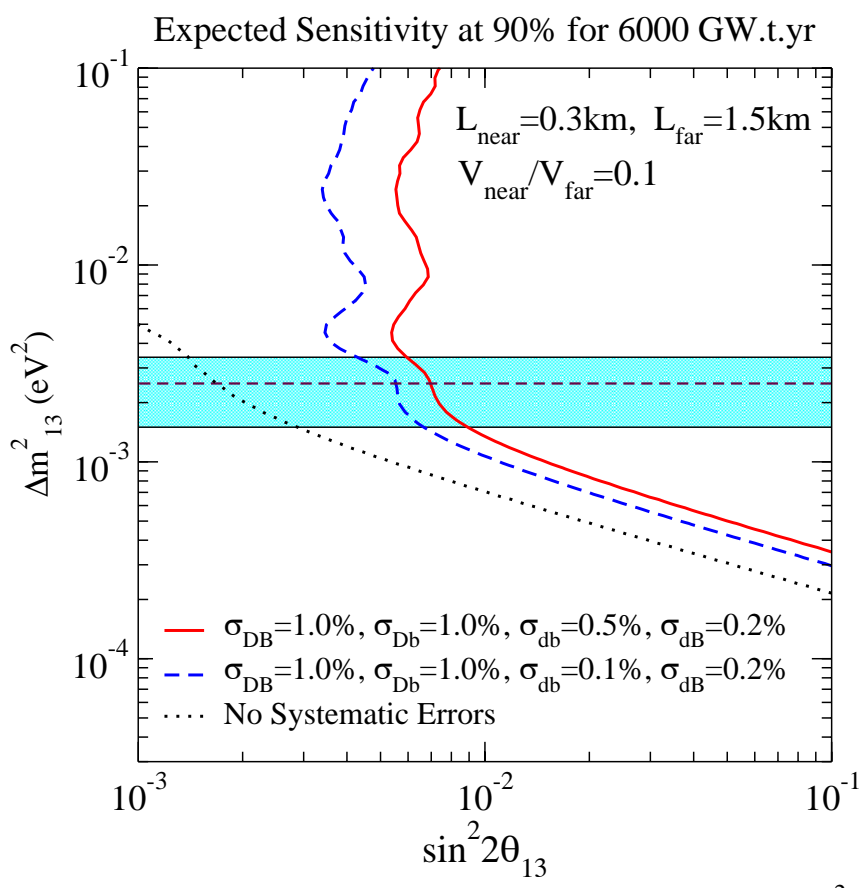

FIG. 6: $90 \% \mathrm{CL}$ sensitivity curve in the parameter space of $\Delta m_{13}^{2}$ and $\sin ^{2} 2 \theta_{13}$ for 3 years of data taking assuming the same systematic errors as in Fig. 5. The blue band indicates the allowed range of $\Delta m_{13}^{2}$ coming from atmospheric neutrino observation [11].

In Fig. 6 we show the expected sensitivity in the parameter space of $\Delta m_{13}^{2}$ and $\sin ^{2} 2 \theta_{13}$ for 3 years of data taking assuming the same systematic errors as in Fig. 5.

\section{THE VERY NEAR DETECTOR}

We are currently developing the very near detector, that will serve as a prototype to test detector elements and performance and also as survey tool for systematic studies. The 


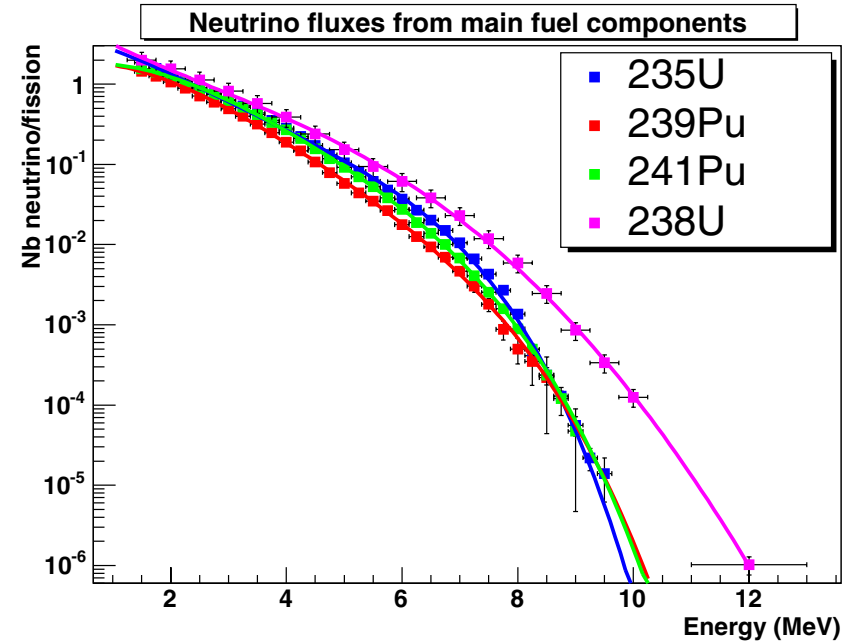

FIG. 7: Measured spectra of antineutrinos from the main components of the nuclear fuel. Taken from Ref. [20].

VND will also be used to monitor the reactor activity, and to provide an additional tool for verification of safeguards and non-proliferation of nuclear weapons.

In fact, the number of antineutrinos detected, $n_{v}$, is related to the reactor thermal power, $W$, by the relationship

$$
n_{v}=\gamma \frac{W N_{H}}{R^{2}}
$$

where $N_{H}$ is the number of hydrogen atoms in the fiducial volume, $R$ is the distance from the source to the detection point and $\gamma$ is a constant which takes into account the contributions to the power output made by the fissile isotopes ${ }^{235} \mathrm{U},{ }^{238} \mathrm{U}$, ${ }^{239} \mathrm{Pu}$ and ${ }^{241} \mathrm{Pu}[16]$.

Recent studies have shown the feasibility of using the antineutrino emission from nuclear reactors as an on-line monitor of the reactor activity. A prototype of this kind of detector is running at the San Onofre reactor (U.S.A.) [17], and a previous experiment was done at the Rovno reactor (Ukraine) [16].

Recent theoretical work shows also that a detector capable of measuring the antineutrino spectrum with high precision can determine not only the thermal power of the reactor but also the fractions of isotopes in the fuel. It is possible to achieve an accuracy of a few percent without knowing the initial fuel compositions [18] because the neutrino energy spectrum from each of the different isotopes is different, as shown in Fig. 7 [19].

In a joint survey of the Angra site with engineers from the Eletronuclear company a preliminary location at $66 \mathrm{~m}$ from the reactor core has been selected for the position of the Very Near Detector. At this distance we expect about 1,000 events/day for a 1 ton detector.

\section{SUMMARY}

We have presented the status and plans of the neutrino oscillation experiment to be performed at the Angra dos Reis nuclear complex in Brazil. This experiment proposes to reach a sensitivity to anti-neutrino disappearance down to $\sin ^{2} 2 \theta_{13} \sim$ 0.006 by measuring the spectrum distortion caused by the oscillation.

As a first step, we are developing a small prototype detector close to the reactor core $(L \simeq 60-70 \mathrm{~m})$, the very near detector, which will also be used to monitor the reactor activity, and to provide an additional tool for verification of safeguards and non-proliferation of nuclear weapons. We are currently performing Monte Carlo simulations to achieve the best detector design and studying solutions to the front-end electronics and data acquisition systems.

We intend to start experimental activities in the Angra site at the end of 2006, measuring the local muon flux, and installing communication systems for remote control and data transfer. The planned turn-on dates are 2008 for the Very Near Detector and 2013 for the complete Angra configuration.

\section{Acknowledgments}

This project is sponsored by the LAMPADIA Foundation, Fundação de Amparo à Pesquisa do Estado de Rio de Janeiro (FAPERJ), Fundação de Amparo à Pesquisa do Estado de São Paulo (FAPESP), and Conselho Nacional de Desenvolvimento Científico e Tecnolgico (CNPq).
[1] F. Reines and C. L. Cowan, Nature, 178, 446 (1956); Phys. Rev. 92, 830 (1953); ibid. 113, 273 (1959); C. L. Cowan et al., Science 124, 103 (1956).

[2] K. Eguchi et al. [KamLAND Collaboration], Phys. Rev. Lett. 90, 021802 (2003); T. Araki et al. [KamLAND Collaboration], Phys. Rev. Lett. 94, 081801 (2005).

[3] L. Wolfenstein, Phys. Rev. D 17, 2369 (1978); S. P. Mikheyev and A. Yu. Smirnov, Yad. Fiz. 42, 1441 (1985) [ Sov. J. Nucl. Phys. 42, 913 (1985)]; Nuovo Cim. C 9, 17 (1986).

[4] M. C. Gonzalez-Garcia and Y. Nir, Rev. Mod. Phys. 75, 345 (2003); S. Pakvasa and J. W. F. Valle, Proc. Indian Natl. Sci. Acad. 70A, 189 (2004); R. N. Mohapatra et al., arXiv:hep$\mathrm{ph} / 0510213$.
[5] J. Burguet-Castell et al., Nucl. Phys. B 608, 301 (2001); H. Minakata and H. Nunokawa, JHEP 0110, 001 (2001); G. Fogli and E. Lisi, Phys. Rev. D 54, 3667 (1996); V. Barger, D. Marfatia and K. Whisnant, Phys. Rev. D 65, 073023 (2002).

[6] K. Anderson et al., arXiv:hep-ex/0402041 (also available at http://www.hep.anl.gov/minos/reactor13/white.html ); See also talks at Fourth Workshop on Future Low Energy Experiments, Hotel do Frade, Angra dos Reis (RJ), Brazil, Feb. 23-25 2005. Talks available at http://www.ifi.unicamp.br/ lenews05/

[7] H. Minakata et al., Phys. Rev. D 68, 033017 (2003) [Erratumibid. D 70, 059901 (2004)]; K. Hiraide et al., Phys. Rev. D 73, 093008 (2006). 
[8] J. C. Anjos et al., Nucl.Phys.B Proc.Suppl., 155, 231 (2006).

[9] M. Aglietta et al., [LVD Collaboration], Phys. Rev. D 58, 092005 (1998).

[10] B. Aharmim et al. [SNO Collaboration], Phys. Rev. C 72, 055502 (2005).

[11] Y. Fukuda et al. [Super-Kamiokande Collaboration], Phys. Rev. Lett. 81, 1562 (1998).

[12] M. H. Ahn et al. [K2K Collaboration], Phys. Rev. Lett. 90, 041801 (2003).

[13] D. G. Michael et al. [MINOS Collaboration], arXiv:hepex/0607088.

[14] E. Kemp, PhD thesis, State University at Campinas - UNI-
CAMP (2000).

[15] H. Minakata and H. Sugiyama, Phys. Lett. B580, 216 (2004).

[16] Yu. V. Klimov et al., Atomic Energy, 73, 123 (1994).

[17] A. Bernstein, Y. F. Wang, G. Gratta and T. West, J. Appl. Phys. 91 4762, (2002) [arXiv:nucl-ex/0108001].

[18] P. Huber and T. Schwetz, Phys. Rev. D 70, 053011 (2004).

[19] K. Schreckenbach et al., Phys. Lett. B 160, 325 (1985); A. A. Hahn et al., Phys. Lett. B218, 365 (1989).

[20] F. Ardellier et al., "Letter of intent for double-CHOOZ: A search for the mixing angle theta(13)," arXiv:hep-ex/0405032. 